Original Article $\quad$ www.pjkd.com.pk

\title{
"PICC"ing Patients with Chronic Kidney Disease
}

\author{
Sonia Yaqub, Muhammad Raheel Abdul Razzaque, Zarghoona Naeem
}

Section of Nephrology,

Department of Medicine,

Aga Khan University,

Stadium Road,

Karachi, Pakistan

\begin{abstract}
:
Patients with Chronic Kidney Disease (CKD) eventually progress to kidney failure and require dialysis. These patients often present with complicated medical conditions requiring intravenous medical therapies; however, at the same time it is of paramount importance to maintain integrity of veins to provide a future hemodialysis (HD) vascular access. Peripherally inserted central catheters (PICC) are used for patients requiring prolonged intravenous medications. PICC lines may cause local trauma and influence the future vascular access creation. The aim of this study was to determine the frequency and indications of PICC lines use in hospitalized patients at a tertiary care hospital.

Patients and Methods: All patients were retrospectively reviewed over 2 months for PICC line placement, indications and their estimated glomerular filtration rate.

Results: Total of 147 PICC lines were placed and $20 \%$ of such patients had an eGFR $<60 \mathrm{ml} / \mathrm{min}$ with possible need of vascular access in future.

Conclusion:

Placement of PICC lines in CKD patients is common and should be discouraged due to the possible need in the future for vascular access.
\end{abstract}

Key Words: PICC line, peripherally inserted central catheter, vascular access, arteriovenous fistula, CKD, hemodialysis.

\author{
Corresponding author: \\ Dr. Sonia Yaqub \\ Assistant professor \\ Director Nephrology Residency Program \\ Department of Medicine \\ Aga Khan University \\ Karachi \\ Email: Sonia.yaqub@aku.edu
}

Received: February 29, 2020. Accepted April 12, 2020.

PJKD 2020;4(2):237-239

\section{Introduction:}

Patients with Chronic Kidney Disease (CKD) are at risk for progression to kidney failure and eventually require dialysis. These patients often present with complicated medical conditions requiring intravenous medical therapies; however, at the same time it is of paramount importance to maintain integrity of veins to provide a future hemodialysis (HD) vascular access. Recent years have seen an expansion in the use of peripherally inserted central catheters (PICC). PICC lines may cause local trauma to vessels and hence may preclude future vascular access creation and its longevity.

The aim of this study was to determine the frequency and indications of PICC lines use in hospitalized patients at a tertiary care hospital.

\section{Methods:}

We conducted a retrospective review of medical records of hospitalized patients who had undergone PICC placement at Aga Khan University between January1st to February 20, 2020. The study was reviewed and exempted by the ethics review committee of the institution. Demographic data on age, gender, baseline eGFR (estimated glomerular filtration rate) and indications of PICC lines was collected. CKD was defined as eGFR of $<60 \mathrm{ml} / \mathrm{min}$ for 3 or more months. Data is described as numbers, mean and percentage

\section{Results:}

A total of 147 patients underwent PICCs. Mean age was 58.4 years; 68\% were males. Almost 20\% $(n=28)$ of patients had underlying CKD and hence were potential candidates for vessels preservation, Figure 1. Figure 2 shows baseline estimated GFR distribution among the study population. The most common indication for PICCs was long-term antibiotics administration (50\% cases) after discharge. 


\section{PICC Lines $n=147$}

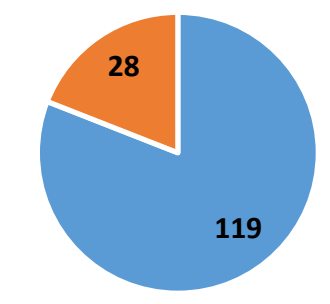

$-\mathrm{eGFR}>60 \| \mathrm{eGFR}<60$

Figure 1: Frequency of patients undergoing PICC lines with or without CKD (eGFR $<60 \mathrm{ml} / \mathrm{min})$

\section{Discussion:}

In our study, we found that almost one fifth of the patient cohort getting PICCs were known to have underlying CKD. It is well recognized that due to multiple and complex coexisting medical problems, patients suffering from CKD are more likely to receive PICCs, potentially increasing their risk for loss of veins.

It is generally perceived that PICCs are safe and effective form of venous access with certain advantages over others such as ease of placement, lower rate of complications, and longevity allowing ease of home administration of drugs. However, a large number of complications associated with PICCs are being recognized increasingly. Besides the increased risk of thromboembolism, PICCs may also cause local trauma to peripheral as well as central veins resulting in central venous stenosis eventually predisposing to future vein failure. ${ }^{2}$ This poses serious implications for patients suffering from CKD who would potentially require a vascular access creation for hemodialysis.

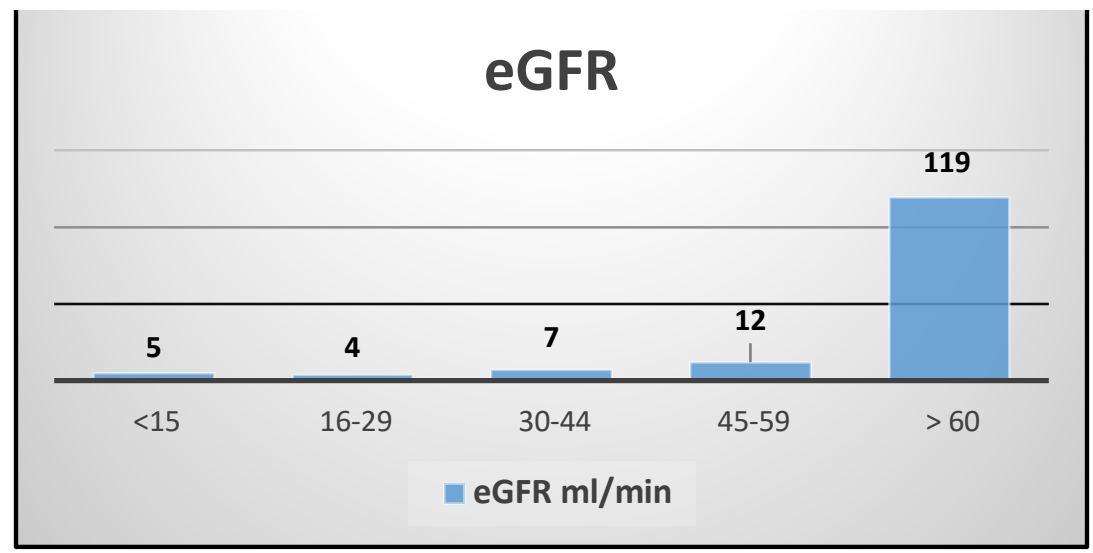

Figure 2. The distribution of various eGFR categories in patients undergoing PICC placement.

When it comes to vascular access for patients requiring $\mathrm{HD}$, the native arterio-venous fistula (AVF) is the access of choice as it is associated with superior patency and lower costs as well as morbidity and need for hospitalization as opposed to synthetic grafts or catheters. Premature exhaustion of veins from use of PICC lines results in failure to create AVF which may lead to prolonged use of HD catheters.

A case-control study of 282 hemodialysis patients by El Ters et al found a strong and independent association between PICC use and lack of a functioning $\mathrm{AVF}^{4}$. Another population - based analysis including 33,918 adult Medicare beneficiaries from the US Renal Data System also supports the findings. In this study PICC placement before or after HD initiation was strongly associated with failure to transition to a working fistula and transition to any permanent access was lower, despite a small increase in the percentage of synthetic grafts. Furthermore, in the same study, PICC placed after HD initiation was associated with a higher risk of death, which remained significant, despite adjustment for various factors.

It is also interesting to note that in the above-mentioned studies, 30\% - 50\% of PICCs were placed for nonspecific indications, such as difficult vascular access. Our study reported similar findings with half of the patients getting PICC lines due to ease of antibiotics administration. 
Considering the adverse outcomes for the CKD patients several societies have prepared guidelines to limit the use of PICCs in general and especially in CKD patients. In 2012, the American Board of Internal Medicine Foundation in partnership with Consumer Reports initiated the Choosing Wisely campaign to identify areas in patient care and resource use with the greatest opportunities for improvement. ${ }^{6}$ An advisory group from the American Society of Nephrology submitted recommendations for inclusion in this campaign. One of the five recommendations of the group was not to place PICCs in patients with CKD stages 3 - 5 without consulting a nephrologist. ${ }^{6}$ The National Kidney Foundation Kidney Disease Outcomes Quality Initiative guidelines for vascular access state that, in patients with CKD stage 4 or 5, forearm and upper arm veins suitable for placement of vascular access should not be used for venipuncture or the placement of intravenous catheters, subclavian catheters, or peripherally inserted central catheter lines. ${ }^{7}$ The Fistula First Breakthrough Initiative (FFBI) also recommends against PICC use in patients at risk for or with known CKD stage 3-5, or end stage renal disease (ESRD) or even if the patient is a renal transplant patient. ${ }^{8}$ The FFBI offers an alternative management strategy: a small bore central catheter (SBCC) in the internal jugular (IJ) vein is recommended. SBCCs can last longer than PICCs, can be easily replaced, and have fewer complications for the period of time needed

When practitioners are faced with whether or not to use a PICC line in a patient with possible CKD, the Fistula First Coalition recommends to:

1. Review each patient' s estimated glomerular filtration rate (eGFR) to identify CKD and/or classify the stage.

2. Obtain a nephrology consult if CKD is present.

3. Use a small-caliber Internal Jugular catheter instead of a PICC line for other intravenous treatment purposes.

4. Consider alternatives to PICC lines whenever possible

Clinicians providing care to patients with CKD should consider potential long - term consequences of the PICCs compared to their shortterm convenience. There is a need to create awareness in the general medical community about careful selection of patients because PICC insertion occurs most commonly in the hospital setting and its use continues unchecked in CKD population due to the perceived ease and the pressure to discharge patients quickly.

It is the responsibility of Nephrologists to remain vigilant in preventing PICC placement in patients with CKD, since a significant number of patients with CKD are under the care of non-nephrologists. Moreover, interventional radiologists placing PICCs should also be educated regarding the necessity to preserve peripheral veins in CKD patients. There is also a need for guidelines and policies at institutional level that require nephrology consultation for any PICC request in patients with CKD may be a good starting point in this respect. Mutual understanding among primary care physicians, nephrologists, nurses, interventionists, and hospital administrators cannot be overemphasized. Where available electronic alerts may facilitate where electronic health records are in use. Last but not the least, patients with CKD and their family and caregivers need to be apprised of the potential risks of PICCs and of need to avoid them.

\section{Conclusion:}

Our study shows that contrary to the guidelines, PICCs are still placed in a significant number of patients with kidney disease. It is the responsibility of nephrologists to educate hospitalists, critical care specialists, internists, patients, and families, about the importance of veins preservation and to promote alternative venous access in CKD population to preserve future venous access.

\section{Conflict of Interest: None Declared}

\section{References:}

1. Chopra V, Flanders SA, Saint S. The problem with peripherally inserted central catheters. JAMA. 2012;308(15):1527-8.

2. Greene MT, Flanders SA, Woller SC, Bernstein SJ, Chopra V. The Association Between PICC Use and Venous Thromboembolism in Upper and Lower Extremities. Am J Med. 2015;128(9):986-93 e1.

3. McGill RL, Tsukahara T, Bhardwaj R, Kapetanos AT, Marcus RJ. Inpatient venous access practices: PICC culture and the kidney patient. J Vasc Access. 2015;16(3):206-10.

4. El Ters M, Schears GJ, Taler SJ, Williams AW, Albright RC, Jenson BM, et al. Association between prior peripherally inserted central catheters and lack of functioning arteriovenous fistulas: a case-control study in hemodialysis patients. Am J Kidney Dis. 2012;60(4):601-8.

5. McGill RL, Ruthazer R, Meyer KB, Miskulin DC, Weiner DE. Peripherally Inserted Central Catheters and Hemodialysis Outcomes. Clin J Am Soc Nephrol. 2016;11(8):1434-40.

6. Williams AW, Dwyer AC, Eddy AA, Fink JC, Jaber BL, Linas SL, et al. Critical and honest conversations: the evidence behind the "Choosing Wisely" campaign recommendations by the American Society of Nephrology. Clin J Am Soc Nephrol. 2012;7(10):1664-72.

7. Vascular Access Work G. Clinical practice guidelines for vascular access. Am J Kidney Dis. 2006;48 Suppl 1:S176-247.

8. Fistula First Breakthrough Initiative: National Vascular Access Improvement Initiative. Available at: http://www.fistulafirst.org/ 